\title{
INFLUÊNCIA DA PRÉ-DEFORMAÇÃO PLÁSTICA, DA TEMPERATURA E DO TEMPO DE AQUECIMENTO NO ENVELHECIMENTO DO AÇO BH220 ATRAVÉS DE PLANEJAMENTO DE EXPERIMENTOS
}

\author{
Rafael Fagundes Ferreira ' \\ Nathalia Sette Câmara Magalhães ' \\ José Rubens Gonçalves Carneiro ${ }^{2}$ \\ Marcello Rosa Dumont ${ }^{3,4}$
}

\section{Resumo}

Aços Bake Hardening são aços laminados a frio com envelhecimento controlado. Estes aços caracterizam-se pela boa conformação e por apresentarem aumento de resistência mecânica devido à deformação e ao envelhecimento por tratamento térmico. Nesse trabalho corpos de prova de tração do material $\mathrm{BH} 220$ foram submetidos à pré-deformações que variam de $0 \%$ a $9 \%$ por tração uniaxial e posteriormente submetidos a tratamento térmico realizado em temperaturas que variam de $140^{\circ} \mathrm{C}$ a $210^{\circ} \mathrm{C}$ com tempos de aquecimento entre 5 minutos e 45 minutos. Foi confirmado que as temperaturas de envelhecimento, na faixa estudada, alteraram a variação do limite de escoamento devido ao fenômeno de difusão que é termicamente ativado. Verificou-se que o tempo de aquecimento, dentro do intervalo testado, não causou grandes variações nos resultados e a deformação foi a variável que maior contribuiu para o aumento de propriedade mecânica.

Palavras-chave: Aço BH220; Bake hardening; Work hardening; Planejamento de experimentos.

\section{INFLUENCE OF PLASTIC PRE DEFORMATION, TEMPERATURE AND HEATING TIME IN THE AGING TREATMENT OF THE BH220 STEEL THROUGH DESIGN OF EXPERIMENTS}

\begin{abstract}
Bake Hardening steels are cold rolled steels with controlled aging. These steels are characterized by a good formability and an increase in the strength due to the aging that occurs during heat treatment. In this study, tensile test specimens of the $\mathrm{BH} 200$ steel were submitted to pre deformations ranging from $0 \%$ to $9 \%$ by uniaxial tension, subsequently they were subjected to an aging heat treatment performed at temperatures varying from $140^{\circ} \mathrm{C}$ to $210^{\circ} \mathrm{C}$, in heating times between 5 minutes and 45 minutes. It has been confirmed that the aging temperatures, in the range studied, modified the yield point due to the phenomenon of diffusion, which is thermally activated. In the results, it is possible to verify that the heating time, within the range tested, it is not significant and the deformation was the largest variable that contributed to the increase of mechanical properties.
\end{abstract}

Keywords: $\mathrm{BH} 220$ steel; Bake hardening; Work hardening; Design of experiments.

\section{INTRODUÇÃO}

As usinas siderúrgicas têm procurado atender aos requisitos da indústria automotiva desenvolvendo aços de elevada resistência mecânica e conformabilidade. Os aços
BH220 são aços endurecíveis por deformação e tratamento térmico permitido a diminuição de espessura da chapa utilizada e consequentemente o peso total dos veículos contribuindo,

'Engenharia de Materiais e Ecologia do Produto, Fiat Chrysler Automobiles, Betim, MG, Brasil. E-mail: marcellodumont@gmail.com 2Programa de Pós-graduação em Engenharia Mecânica, Pontifícia Universidade Católica de Minas Gerais - PUC-MG, Belo Horizonte, MG, Brasil. ${ }^{3}$ Departamento de Engenharia de Materiais, Centro Federal de Educação Tecnológica de Minas Gerais - CEFET-MG, Belo Horizonte, MG, Brasil. ${ }^{4}$ Universidade Federal de Minas Gerais - UFMG, Belo Horizonte, MG, Brasil. 
assim, para redução do consumo de combustível e emissão de poluentes. Este aumento da resistência mecânica é conhecido como efeito bake hardening (Efeito $\mathrm{BH}$ ) [I]. $\mathrm{O}$ acréscimo de resistência mecânica nesse aço $\mathrm{BH} 220$ se deve ao envelhecimento estático após estampagem e tratamento térmico em temperatura de $170^{\circ} \mathrm{C}$ durante 20 minutos durante processo de pintura [2-4]. Esse efeito BH é ligado à presença de carbono em solução sólida na matriz ferrítica o que dificulta a movimentação das discordâncias e possibilita interação dessas discordâncias com esses átomos em solução sólida [5-7]. Durante a estampagem da peça, ocorre elevação do número de discordâncias por unidade de volume do material. Posteriormente, no tratamento térmico, pode haver interação do carbono em solução sólida com as discordâncias criadas na estampagem. Deslizamentos dessas discordâncias criadas exigirão maior tensão o que evidencia aumento da resistência mecânica do material [8]. Segundo Montgomery e Runger [9] as técnicas de planejamento e análise de experimentos também são utilizadas para melhorar a qualidade de processos de fabricação com reduzido número de testes.

Este trabalho estuda simultaneamente as influências, sob o efeito $\mathrm{BH}$, da deformação plástica na estampagem e da temperatura e tempo no tratamento térmico no aço $\mathrm{BH} 220$ utilizado para confecção de painéis externos automotivos. O efeito BH é a somatória do endurecimento por tratamento térmico (chamado de $\mathrm{BH}_{\mathrm{n}}$ ) com o endurecimento por deformação (chamado de work hardening $-\mathrm{WH}_{\mathrm{n}}$ ) onde o índice $\mathrm{n}$ representa a deformação inicial antes do tratamento térmico [I].

\section{MATERIAIS E MÉTODOS}

\section{I Análises Química e Metalográfica}

O material utilizado nos ensaios foi uma chapa de aço BH $220 \mathrm{com} 0,7 \mathrm{~mm}$ de espessura com extrabaixo teor de carbono, manganês, alumínio e fósforo, conforme norma FIAT 52814 [10]. Teores de carbono e nitrogênio nessa faixa propiciam envelhecimento por deformação [I I]. As amostras retiradas para análise química têm seção de $42 \mathrm{~mm} \times 42 \mathrm{~mm}$ e foram lixadas com óxido de alumínio em granulometria de 40 mesh. A análise dos elementos Mn, Al, $\mathrm{Si}, \mathrm{P}, \mathrm{Ti}$ e Nb foi obtida por espectrometria de emissão ótica (modo spark) no equipamento SPECTROMAXx LMMI5 da Spectro. $A$ análise dos elementos $C$ e $S$ foi feita em equipamento Leco CS444LS, enquanto que $\mathrm{N}$ foi analisado no Leco TC436. As amostras para análise metalográfica foram cortadas nas dimensões de $10 \mathrm{~mm} \times 20 \mathrm{~mm}$ em uma máquina cut-off, utilizando óleo lubrificante Tromus $B$ (solução aquosa de $5 \%$ ). Estas amostras foram embutidas em baquelite em prensa Leco PR 32, dotada de sistema de aquecimento. A temperatura atingiu $150^{\circ} \mathrm{C}$ com pressão de $2 \mathrm{Kgf} / \mathrm{mm}^{2}$ por 12 minutos. Em seguida estas amostras foram polidas em lixas com granulação 100, 150, 220,
$320,400,600,800,1000$ e 1200 mesh, refrigeradas com água. Após o polimento mecânico, foi feito o polimento de acabamento em politrizes impregnadas com pasta diamante de $6 \mu \mathrm{m}, 3 \mu \mathrm{m}$ e $0,5 \mu \mathrm{m}$. Posteriormente, as amostras foram atacadas com solução de nital $4 \%$ por tempo entre $10 \mathrm{se}$ $30 \mathrm{~s}$. A classificação das inclusões não metálicas foi realizada utilizando microscópio ótico, marca Zeiss Axioplan, com aumento de 100 vezes. Para determinação do tamanho de grão foi utilizado o equipamento Leica Quantimet $600 \mathrm{HR}$ composto por microscópio Polyvarmet Reichert-jung, módulo de câmera de vídeo N50 e um computador com o programa Quantimet $600 \mathrm{QWin}$.

\subsection{Ensaios Mecânicos de Tração}

As propriedades mecânicas foram obtidas por ensaio de tração uniaxial em máquina universal de ensaios Instron modelo $4467 \mathrm{H} 2000$ com capacidade de $30 \mathrm{kN}$. Os corpos de prova foram confeccionados na direção transversal ao sentido de laminação da chapa, conforme norma FIAT $528 / 4$ [ I0]. Esses corpos de prova foram usinados conforme norma FIAT 50113 [12]. As deformações iniciais foram geradas com velocidade constante de $1,2 \mathrm{~mm} / \mathrm{min}$ até atingir os valores previstos. Na sequência os corpos de prova foram submetidos ao tratamento térmico em diferentes temperaturas e tempo de permanência em estufa. Após os tratamentos térmicos os corpos de prova foram ensaiados até ruptura segundo norma UNI EN I0002-I [I3]. Os valores do limite de escoamento (LE), limite de resistência (LR), e alongamento foram calculados, conforme norma ASTM A-370 [14]. Também foram ensaiados cinco (5) corpos de prova sem deformação e sem tratamento térmico tomando estes resultados de propriedades mecânicas como referência para o material no estado de fornecimento.

O ganho de resistência mecânica referente ao tratamento térmico em uma determinada deformação $n \%$, $\mathrm{BH}_{\mathrm{n}}$, é calculado pela diferença entre os valores de limite de escoamento antes e após o tratamento térmico. $O$ ganho de resistência mecânica gerado por um determinado valor de encruamento $\mathrm{n} \%, \mathrm{WH}_{\mathrm{n}}$, é calculado pela diferença entre os valores de limite de escoamento antes e após a deformação. A Figura I mostra esquematicamente o método de obtenção dos efeitos $\mathrm{BH}$ e WH para um aço pré-deformado em $2 \%$ $\left(\mathrm{BH}_{2} \mathrm{eWH}_{2}\right)$ e envelhecido por uma temperatura de $170^{\circ} \mathrm{C}$ por 20 minutos [15].

\subsection{Planejamento do Experimento}

Neste trabalho foi escolhido o planejamento fatorial completo tipo composto central com três fatores e dois níveis $\left(2^{3}\right)$ para modelar e analisar a influência de cada variável sobre o efeito $\mathrm{BH}$ utilizando o software Minitab ${ }^{\circledR}$. Foi estipulado um valor intermediário entre o nível central e os níveis superior e inferior, chamada de alfa, com valor de $+0,5$ e - 0,5 . Correspondente a um determinado deslocamento sobre o eixo que neste caso representa o ponto médio entre 


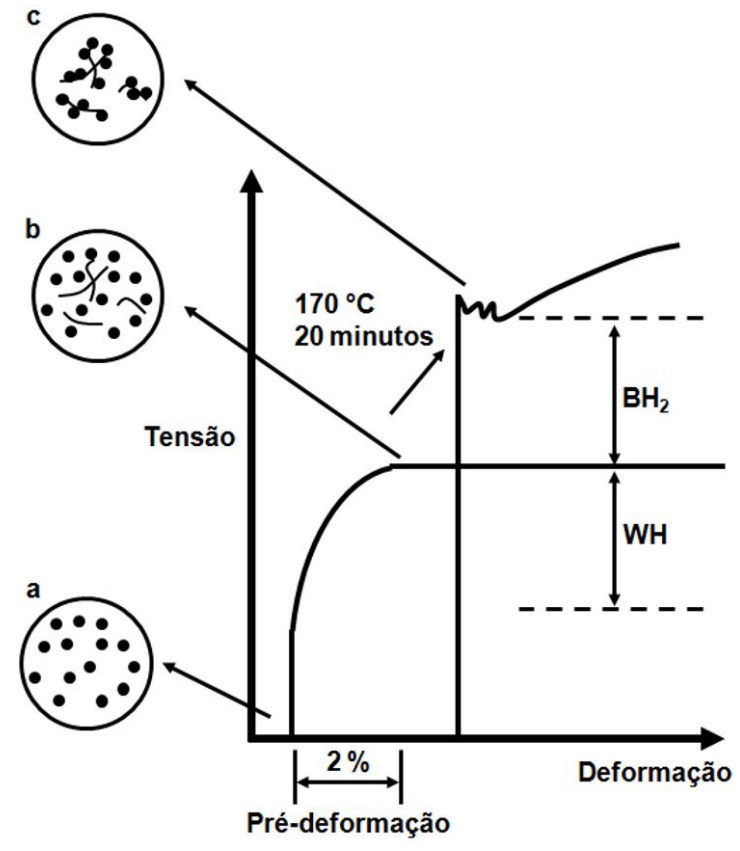

Figura I. Método de obtenção do efeito $\mathrm{BH}_{2}$ e $\mathrm{WH}_{2}$ em ensaio de tração pela curva tensão VS deformação. Em cada etapa, são indicados: os átomos de $\mathrm{C}$ em solução (a), discordâncias geradas na deformação (b) e discordâncias imobilizadas após tratamento térmico (c), adaptado de Lemos [15]. o nível central e os níveis superior e inferior [9]. As variáveis independentes, fatores, definidas foram o percentual de deformação, a temperatura e o tempo de permanência na estufa. Foram utilizadas deformações no intervalo de $0 \%$ a $9 \%$ em tração, temperatura de envelhecimento entre $140^{\circ} \mathrm{C}$ a $210^{\circ} \mathrm{C}$ e o tempo de 5 min a $45 \mathrm{~min}$. Estes valores compreendem os valores tipicamente utilizados na indústria automotiva para painéis externos e para o processo de pintura da carroceria [2-4] com ligeira extrapolação no tempo e temperatura. Os níveis centrais foram deformação de $4,5 \%$, temperatura de envelhecimento de $175^{\circ} \mathrm{C}$ e o tempo de $25 \mathrm{~min}$. Os valores gerados pelo alfa foram deformações de $2,25 \%$ e $6,75 \%$, temperaturas de $157,5^{\circ} \mathrm{C}$ e $192,5^{\circ} \mathrm{C}$ e tempos de $15 \mathrm{~min}$ e $35 \mathrm{~min}$. Os níveis superior, central e inferior para os fatores são mostrados na Tabela I.

Foram geradas 15 combinações de fatores que em triplicata geraram 45 amostras as quais estão descritas na Tabela 2.

Foi utilizado como variável dependente ou de resposta o valor de efeito $\mathrm{BH}$ calculado com os dados de limite de escoamento no ensaio de tração. A análise foi realizada com nível de confiança de $95 \%$, utilizando o modelo de interação full quadratic que abrange todas as interações entre os fatores. $O$ modelo de regressão para este tipo de planejamento fatorial $2^{3}$ utilizado foi através da metodologia de superfície de resposta (MSR) considerando

Tabela I. Fatores e níveis do planejamento de experimentos

\begin{tabular}{lccccc}
\hline \multicolumn{1}{c}{ Fator } & Nível sup. + I & Alfa $+\mathbf{0 , 5}$ & Central 0 & Alfa -0,5 & Nível Inf. - I \\
\hline Tempo $(\min )$ & 45 & 35 & 25 & 15 & 5 \\
Temperatura $\left({ }^{\circ} \mathrm{C}\right)$ & 210 & 192,5 & 175 & 157,5 & 140 \\
Deformação $(\%)$ & 9 & 6,75 & 4,5 & 2,25 & 0 \\
\hline
\end{tabular}

Tabela 2. Amostras geradas no planejamento de experimentos e seus fatores

\begin{tabular}{|c|c|c|c|c|c|c|}
\hline Ensaios & & Amostra & & $\begin{array}{c}\text { Temperatura } \\
\left({ }^{\circ} \mathrm{C}\right)\end{array}$ & $\begin{array}{c}\text { Tempo } \\
\text { (min) }\end{array}$ & $\begin{array}{c}\text { Deformação } \\
(\%)\end{array}$ \\
\hline $\mathrm{I}, 2,3$ & 1.1 & 1.2 & 1.3 & 140,0 & 5 & 0,00 \\
\hline $4,5,6$ & 2.1 & 2.2 & 2.3 & 210,0 & 5 & 0,00 \\
\hline $7,8,9$ & 3.1 & 3.2 & 3.3 & 140,0 & 45 & 0,00 \\
\hline $10,11,12$ & 4.1 & 4.2 & 4.3 & 210,0 & 45 & 0,00 \\
\hline $13,14,15$ & 5.1 & 5.2 & 5.3 & 140,0 & 5 & 9,00 \\
\hline $16,17,18$ & 6.1 & 6.2 & 6.3 & 210,0 & 5 & 9,00 \\
\hline $19,20,21$ & 7.1 & 7.2 & 7.3 & 140,0 & 45 & 9,00 \\
\hline $22,23,24$ & 8.1 & 8.2 & 8.3 & 210,0 & 45 & 9,00 \\
\hline $25,26,27$ & 9.1 & 9.2 & 9.3 & 157,5 & 25 & 4,50 \\
\hline $28,29,30$ & 10.1 & 10.2 & 10.3 & 192,5 & 25 & 4,50 \\
\hline $31,32,33$ & 11.1 & 11.2 & 11.3 & 175,0 & 15 & 4,50 \\
\hline $34,35,36$ & 12.1 & 12.2 & 12.3 & 175,0 & 35 & 4,50 \\
\hline $37,38,39$ & 13.1 & 13.2 & 13.3 & 175,0 & 25 & 2,25 \\
\hline $40,41,42$ & 14.1 & 14.2 & 14.3 & 175,0 & 25 & 6,75 \\
\hline $43,44,45$ & 15.1 & 15.2 & 15.3 & 175,0 & 25 & 4,50 \\
\hline
\end{tabular}


um modelo de segunda ordem que contem os três fatores. A dependência da resposta, $y$, sobre os fatores é mostrada na Equação I $[9,16]$.

$$
y=\beta_{0}+\sum_{i=1}^{k} \beta_{i} x_{i}+\sum_{i=1}^{k} \beta_{i i} x_{i}^{2}+\sum_{i<j} \sum \beta_{i j} x_{i} x_{j}+\varepsilon
$$

onde $\beta_{0}$ é um termo constante, $\beta_{\mathrm{i}}$, $\beta_{\mathrm{ii}}$ e $\beta_{\mathrm{ij}}$ são os coeficientes, $\varepsilon$ é o erro, $x_{i}$ e $x_{i}$ são as variáveis (temperatura, tempo, deformação) e k é o número de variáveis (3).

O modelo ajustado de segunda ordem pela MSR é mostrado na Equação 2 [9].

$$
\hat{y}=\hat{\beta}_{0}+\sum_{i=1}^{k} \hat{\beta}_{i} x_{i}+\sum_{i=1}^{k} \hat{\beta}_{i i} x_{i}^{2}+\sum_{i<j} \sum \hat{\beta}_{i j} x_{i} x_{j}+\varepsilon
$$

onde $\hat{\beta}$ denota a estimativa de mínimos quadrados de $\beta$.

Três amostras em posições aleatórias no gráfico de superfície foram escolhidas e ensaiadas para se observar a aproximação dos dados previstos aos encontrados.

\section{RESULTADOS E DISCUSSÃO}

$\mathrm{O}$ aço $\mathrm{BH} 220$ apresentou valores extrabaixo de elementos intersticiais $\mathrm{C}$ e $\mathrm{N}$ com presença de elementos substitucionais $\mathrm{Mn}$ e $\mathrm{P}$ para ajuste de propriedades mecânicas, em conformidade com o especificado [8]. A Tabela 3 mostra a composição química obtida do aço $\mathrm{BH} 220$ utilizado nos experimentos.

A microestrutura desse aço no estado de fornecimento (laminado a quente e recozido) para espessura de $0,7 \mathrm{~mm}$ evidenciou a presença de ferrita com tamanho de grão de $9 \mu \mathrm{m}(+/-\mathrm{I} \mu \mathrm{m})$, mostrado na Figura 2.

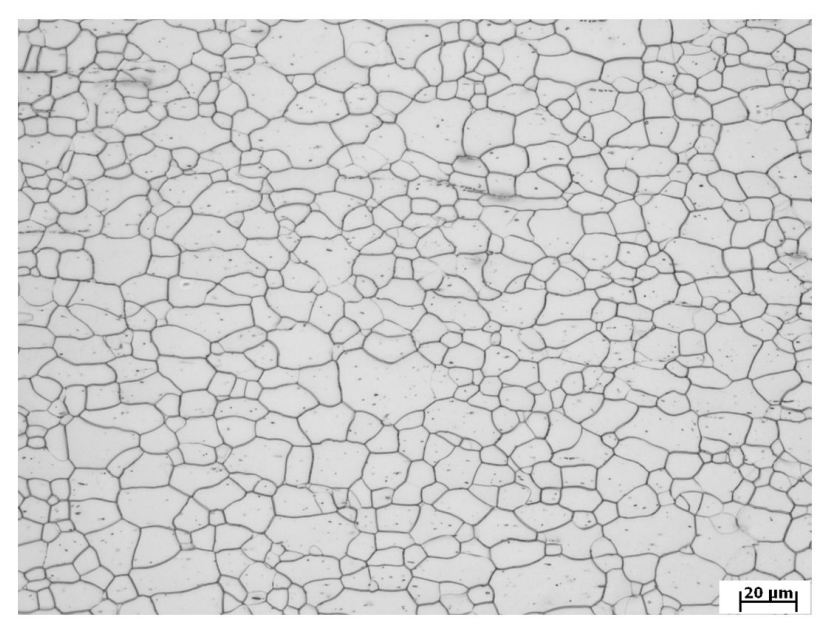

Figura 2. Microestrutura do aço $\mathrm{BH} 220$ evidenciando a presença de ferrita com granulação fina.
No estado de fornecimento foi encontrado limite de escoamento de $237 \mathrm{MPa}$ (+/- $2 \mathrm{MPa})$, limite de resistência de $355 \mathrm{MPa}$ (+/- I MPa) e alongamento de 38\% (+/- 1\%). Estas propriedades mecânicas estão mostradas na Tabela 4.

A Tabela 5 mostra os resultados individuais encontrados para os valores de $\mathrm{WH}_{n}, \mathrm{BH}_{n}$ e efeito $\mathrm{BH}\left(\mathrm{WH}_{n}+\mathrm{BH}_{n}\right)$ para os experimentos realizados nas diferentes deformações, tempos e temperaturas de envelhecimento. Os valores apresentam erro típico de $1 \%$.

Foram obtidos valores de efeito $\mathrm{BH}$ entre 0,5 $\mathrm{MPa}$ (amostra I.I) e 120,5 MPa (amostra 8. I). Segundo Kuang et al. [ 17] valores de efeito $\mathrm{BH}$ entre aproximadamente 20 $\mathrm{MPa}$ e $60 \mathrm{MPa}$ são devido ao travamento das discordâncias pelos átomos intersticiais de carbono e nitrogênio.

O modelo de mínimos quadrados obtido para o efeito $\mathrm{BH}$ [9] foi $\hat{y}=-35+0,1 x_{1}-0,2 x_{2}+30 x_{3}+0,001 x_{1}^{2}+$ $0,004 x_{2}^{2}-1,2 x_{3}^{2}+0,0007 x_{1} x_{2}-0,049 x_{2} x_{3}-0,005 x_{1} x_{3}$. A análise de variança para este modelo é mostrada na Tabela 6 .

O modelo mostra que o fator que mais contribuiu para o efeito $\mathrm{BH}$ foi a deformação. Os demais fatores geram pequenas influências. $O$ coeficiente de determinação $\left(R^{2}\right)$ encontrado foi de $\mathrm{R}^{2}=99,7 \%$ e $\mathrm{R}_{\text {ajust. }}=99,7 \%$ sugerindo que $99 \%$ da variabilidade dos dados é considerada pelo modelo [9].

A Figura 3 mostra os gráficos de superfícies para o modelo do efeito $\mathrm{BH}$, mostrando as interações de deformação em função do tempo com a temperatura fixa no ponto central $\left(175^{\circ} \mathrm{C}\right)$ e deformação em função da temperatura com tempo fixo no ponto central (25 min).

Foi observado que a elevação do tempo praticamente não propiciou aumentos nos valores de efeito $\mathrm{BH}$. Acréscimos na temperatura e principalmente na deformação provocam valores mais expressivos efeito $\mathrm{BH}$. Recentemente Tang et al. [18] reportaram que o efeito $\mathrm{BH}$ é aumentado com o aumento do tempo e da temperatura de tratamento térmico. Neste caso os resultados encontrados sugerem que o tempo mínimo de 5 min utilizado foi suficiente para promover as alterações estruturais relacionadas ao travamento das discordâncias. $\mathrm{O}$ valor máximo de efeito $\mathrm{BH}$ levantando pelo modelamento matemático foi de $120,5 \mathrm{MPa}(+/-0,4$ $\mathrm{MPa}$ ) com deformação de $8 \%$, temperatura de $210^{\circ} \mathrm{C}$ e tempo de $45 \mathrm{~min}$. Corresponde a aumento de $51 \%$ do limite de escoamento em relação ao material no estado de fornecimento. Com tempo de 5 min e mantendo a deformação e temperatura em $8 \%$ e $210^{\circ} \mathrm{C}$ o valor de efeito $\mathrm{BH}$ sugerido pelo modelamento foi de 1 I 7,0 $\mathrm{MPa}$ (+/- 0,4 MPa).

A análise dos resíduos, mostrada na Figura 4, sugere que os erros possuem distribuição normal e padrão de comportamento satisfatório [9], não revelando anormalidades [16].

Tabela 3. Análise química encontrada no aço utilizado nos experimentos em percentagem em peso

\begin{tabular}{ccccccccc}
\hline $\mathbf{C}$ & $\mathbf{M n}$ & $\mathbf{A l}$ & $\mathbf{S i}$ & $\mathbf{P}$ & $\mathbf{S}$ & $\mathbf{T i}$ & $\mathbf{N b}$ & $\mathbf{N}$ \\
\hline 0,0019 & 0,70 & 0,056 & 0,01 & 0,040 & 0,009 & 0,002 & 0,007 & 0,001 \\
\hline
\end{tabular}


Tabela 4. Resultados obtidos de propriedades mecânicas do aço $\mathrm{BH} 220$ no estado de fornecimento

\begin{tabular}{ccc}
\hline $\begin{array}{c}\text { Limite de } \\
\text { escoamento (MPa) }\end{array}$ & $\begin{array}{c}\text { Limite de } \\
\text { resistência (MPa) }\end{array}$ & $\begin{array}{c}\text { Alongamento } \\
(\%)\end{array}$ \\
\hline $237 \pm 2$ & $355 \pm 1$ & $38 \pm 1$ \\
\hline
\end{tabular}

As três amostras escolhidas com posições aleatórias na superfície foram: deformação, temperatura e tempo, respectivamente, A) $0 \%, 210^{\circ} \mathrm{C}$ e $40 \mathrm{~min}$; B) $2 \%, 210^{\circ} \mathrm{C}$ e $20 \mathrm{~min}$; C) $8 \%, 200^{\circ} \mathrm{C}, 40 \mathrm{~min}$. A Tabela 7 mostra os resultados obtidos de cada conjunto de contraprova, os

Tabela 5. Resultados, em MPa, obtidos de $\mathrm{WH}_{n}, \mathrm{BH}_{n}$ e $\sum\left(\mathrm{WH}_{n}+\mathrm{BH}_{n}\right)$ para o aço $\mathrm{BH} 22 \mathrm{O}$ em condições distintas de deformação, temperatura e tempo

\begin{tabular}{|c|c|c|c|c|c|c|c|c|c|c|c|}
\hline Am. & $\mathbf{W} \mathbf{H}_{n}$ & $\mathrm{BH}_{\mathrm{n}}$ & $\Sigma$ & Am. & $\mathbf{W H}_{\mathrm{n}}$ & $\mathrm{BH}_{\mathrm{n}}$ & $\sum$ & Am. & $\mathbf{W} \mathbf{H}_{\mathbf{n}}$ & $\mathrm{BH}_{\mathrm{n}}$ & $\Sigma$ \\
\hline I.I & 0 & 0 & 0 & 1.2 & 0 & 2 & 2 & 1.3 & 0 & 2 & 2 \\
\hline 2.1 & 0 & 38 & 38 & 2.2 & 0 & 41 & 41 & 2.3 & 0 & 39 & 39 \\
\hline 3.1 & 0 & 4 & 4 & 3.2 & 0 & 5 & 5 & 3.3 & 0 & 5 & 5 \\
\hline 4.1 & 0 & 43 & 43 & 4.2 & 0 & 48 & 48 & 4.3 & 0 & 43 & 43 \\
\hline 5.1 & 91 & 18 & 110 & 5.2 & 90 & 18 & 108 & 5.3 & 90 & 18 & 108 \\
\hline 6.1 & 92 & 27 & 119 & 6.2 & 90 & 26 & 116 & 6.3 & 90 & 25 & 114 \\
\hline 7.1 & 91 & 20 & 111 & 7.2 & 90 & 19 & 109 & 7.3 & 90 & 20 & 110 \\
\hline 8.1 & 91 & 30 & 120 & 8.2 & 90 & 28 & 118 & 8.3 & 89 & 30 & 120 \\
\hline 9.1 & 63 & 24 & 87 & 9.2 & 61 & 26 & 87 & 9.3 & 62 & 27 & 89 \\
\hline 10.1 & 63 & 30 & 94 & 10.2 & 62 & 30 & 92 & 10.3 & 61 & 31 & 92 \\
\hline II.I & 64 & 26 & 89 & 11.2 & 62 & 28 & 90 & 11.3 & 64 & 26 & 90 \\
\hline 12.1 & 63 & 28 & 91 & 12.2 & 62 & 29 & 91 & 12.3 & 62 & 28 & 90 \\
\hline 13.1 & 33 & 27 & 60 & 13.2 & 34 & 29 & 63 & 13.3 & 34 & 31 & 65 \\
\hline 14.1 & 78 & 25 & 104 & 14.2 & 79 & 24 & 103 & 14.3 & 80 & 26 & 106 \\
\hline 15.1 & 62 & 27 & 90 & 15.2 & 62 & 29 & 91 & 15.3 & 62 & 30 & 92 \\
\hline
\end{tabular}

Tabela 6. Análise de variança para o modelo quadrático com o efeito $\mathrm{BH}$ como parâmetro de saída

\begin{tabular}{|c|c|c|c|c|c|c|}
\hline Variável independente & $\begin{array}{c}\text { Estimativa dos } \\
\text { coeficientes }\end{array}$ & & $\begin{array}{l}\text { Erro padrão do } \\
\text { coeficiente }\end{array}$ & T Para $\mathrm{H}_{0}:$ coef $=0$ & & Valor $\mathbf{P}$ \\
\hline Constantes & $-35,4484$ & & 68,1951 & $-0,520$ & & 0,607 \\
\hline Temperatura & 0,1004 & & 0,8196 & 0,123 & & 0,903 \\
\hline Tempo & $-0,2355$ & & 0,3760 & $-0,626$ & & 0,535 \\
\hline Deformação & 29,8729 & & 1,3726 & 21,763 & & 0,000 \\
\hline Temperatura*Temperatura & 0,0012 & & 0,0023 & 0,525 & & 0,603 \\
\hline Tempo*Tempo & 0,0044 & & 0,0072 & 0,614 & & 0,544 \\
\hline Deformação*'Deformação & $-1,2392$ & & 0,1416 & $-8,75 \mid$ & & 0,000 \\
\hline Temperatura*Tempo & 0,0007 & & 0,0006 & I,046 & & 0,303 \\
\hline Temperatura*Deformação & $-0,0488$ & & 0,0028 & $-|7,56|$ & & 0,000 \\
\hline Tempo*Deformação & $-0,0045$ & & 0,0049 & $-0,935$ & & 0,357 \\
\hline Fonte & $\mathbf{G L}$ & SQ seq. & SQ aj. & MQ aj. & $\mathbf{F}$ & $\mathbf{P}$ \\
\hline Regressão & 9 & 61979,2 & 61979,2 & 6886,57 & 1498,69 & 0,000 \\
\hline Linear & 3 & $55765, I$ & $74 I I, 5$ & 2470,51 & 537,65 & 0,000 \\
\hline Temperatura & I & 3353,7 & 0,1 & 0,07 & 0,02 & 0,903 \\
\hline Tempo & 1 & 62,7 & 1,8 & 1,80 & 0,39 & 0,535 \\
\hline Deformação & 1 & 52348,7 & 2176,4 & 2176,42 & 473,64 & 0,000 \\
\hline Quadrado & 3 & 4787,9 & 4787,9 & 1595,98 & 347,32 & 0,000 \\
\hline Temperatura*Temperatura & I & 4354,2 & I,3 & 1,27 & 0,28 & 0,603 \\
\hline Tempo*Tempo & I & 81,8 & 1,7 & 1,73 & 0,38 & 0,544 \\
\hline Deformação*Deformação & I & 351,9 & 351,9 & 351,92 & 76,59 & 0,000 \\
\hline Interação & 3 & 1426,2 & 1426,2 & 475,39 & 103,46 & 0,000 \\
\hline Temperatura*Tempo & 1 & 5,0 & 5,0 & 5,02 & 1,09 & 0,303 \\
\hline Temperatura*Deformação & I & $14|7|$, & $14|7|$, & $|4| 7,||$ & 308,40 & 0,000 \\
\hline Tempo*Deformação & 1 & 4,0 & 4,0 & 4,02 & 0,87 & 0,357 \\
\hline Erro Residual & 33 & $15 \mid, 6$ & $15 \mid, 6$ & 4,60 & & \\
\hline Total & 44 & 62131,7 & & & & \\
\hline
\end{tabular}


A

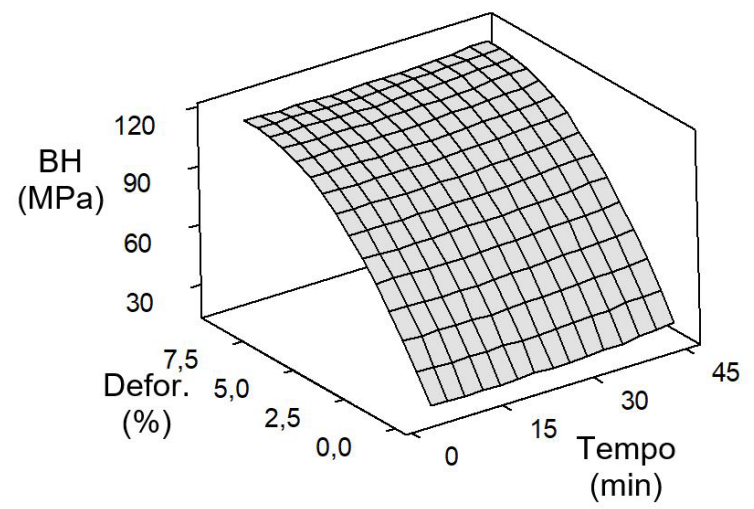

B

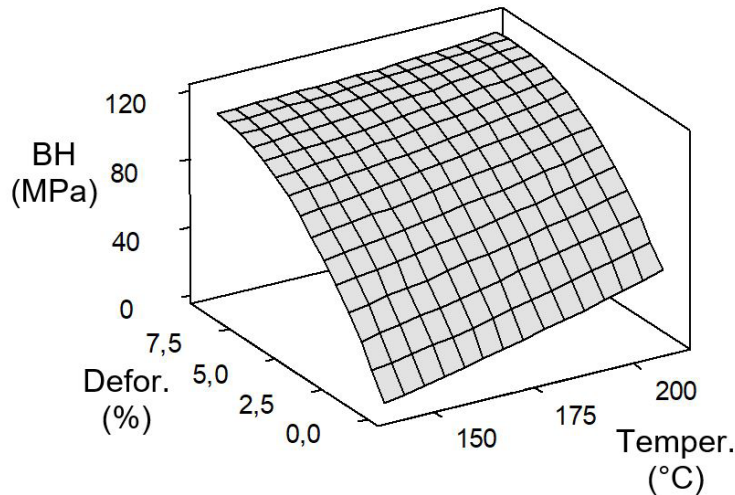

Figura 3. Gráfico de superfície obtido para (A) deformação vs tempo com temperatura de $175^{\circ} \mathrm{C}$ e (B) deformação vs temperatura com tempo de $25 \mathrm{~min}$.
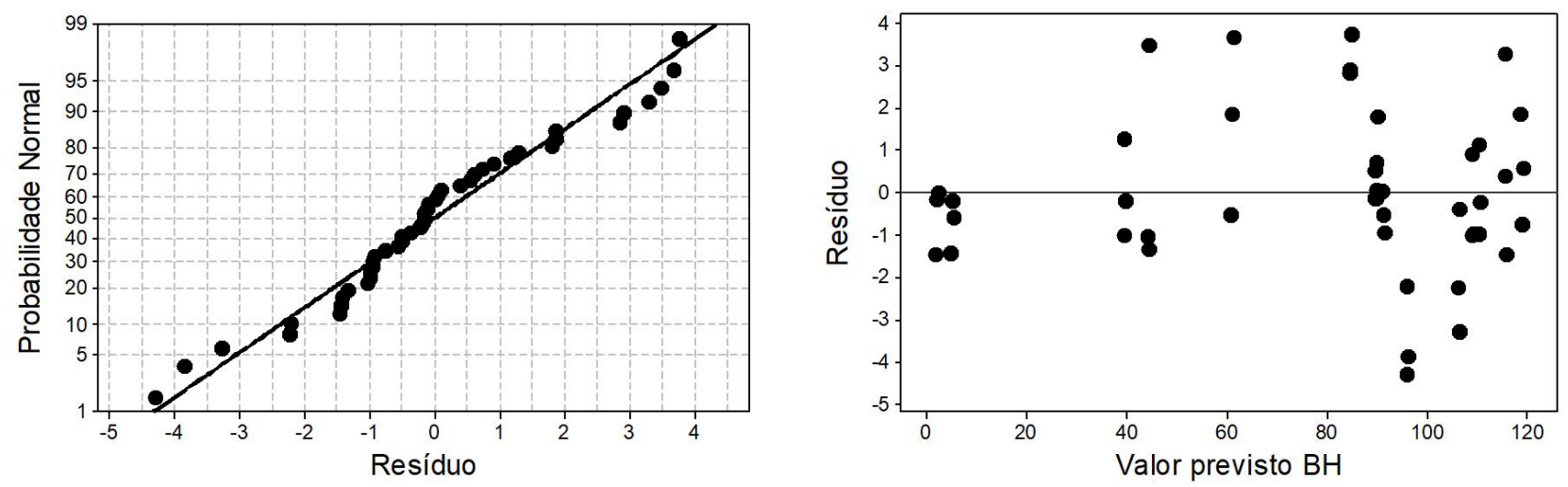

Figura 4. Gráfico de probabilidade normal dos resíduos (esq.) e gráfico de resíduos VS valores previstos para o efeito $\mathrm{BH}$ (dir.).

Tabela 7. Resultados encontrados de $\mathrm{WH}_{\mathrm{n}}+\mathrm{BH}_{\mathrm{n}}(\mathrm{MPa})$ para as amostras de contraprova

\begin{tabular}{|c|c|c|c|}
\hline \multirow{3}{*}{ Amostra } & A) & B) & C) \\
\hline & $0 \% 210^{\circ} \mathrm{C}$ & $2 \% 210^{\circ} \mathrm{C}$ & $8 \% 200^{\circ} \mathrm{C}$ \\
\hline & $35 \mathrm{~min}$ & $20 \min$ & $40 \mathrm{~min}$ \\
\hline CP I & 48 & 70 & 112 \\
\hline CP 2 & 42 & 71 & 112 \\
\hline CP 3 & 46 & 70 & 112 \\
\hline CP 4 & 44 & 71 & 112 \\
\hline CP 5 & 44 & --- & 114 \\
\hline Média & 45 & 70 & 112 \\
\hline Desvio & 2 & 1 & 1 \\
\hline Superfície de resposta & 42 & 74 & 117 \\
\hline Diferença & 3 & 4 & 5 \\
\hline
\end{tabular}

valores previstos de $\mathrm{WH}_{\mathrm{n}}+\mathrm{BH}_{\mathrm{n}}$ conforme os gráficos de superfícies e a diferença entre o resultado dos dois métodos.

A diferença entre o valor modelado pela MSR e o valor médio encontrado para o efeito $\mathrm{BH}$ das amostras ensaiadas situou no intervalo de $3 \mathrm{MPa}$ a $5 \mathrm{MPa}$ com desvio padrão entre I MPa e $2 \mathrm{MPa}$. As superfícies geradas sugerem uma boa aproximação com os valores encontrados experimentalmente.

\section{CONCLUSÕES}

O planejamento de experimentos fatorial com metodologia de superfície de resposta permitiu observar, em uma ampla faixa de valores, diversas zonas de aumento de resistência mecânica, medidos através do efeito $\mathrm{BH}$. As superfícies mostraram resultados de efeito $\mathrm{BH}$ em vários níveis permitindo selecionar os fatores de modo a obter determinados resultados. Quanto maior o valor de $\mathrm{WH}+\mathrm{BHn}$, melhor será a resistência do componente às solicitações mecânicas após processo de fabricação de um componente. $\mathrm{O}$ estudo realizado indica, portanto, que aumentando as deformações obtidas durante a conformação dos painéis maior será o efeito $\mathrm{BH}$ final. Entretanto, em baixas deformações é possível também obter, para o material $\mathrm{BH} 220$, bons resultados de acordo com os valores 
do tratamento térmico, mesmo sendo pequena a influencia deste parâmetro. Maiores temperaturas geram maiores resultados de escoamento, por outro lado, isto implica em aumento do custo do processo. Em relação ao tempo de permanência no tratamento térmico o estudo evidenciou que está variável praticamente não causa variação no resultado de escoamento final dentro dos limites testados. $\bigcirc$ emprego de materiais de maior resistência mecânica permite, para um mesmo desempenho, a redução de espessura no material com consequente redução de peso da estrutura e trazendo como resultado a possibilidade de redução de custo.

\section{Agradecimentos}

Os autores agradecem ao CEFET-MG, à Fiat Automóveis e à PUC-MG pelo suporte técnico e financeiro a este trabalho.

\section{REFERÊNCIAS}

I Ferreira FR, Ferreira JL, Silva SM, Faria ER, Bustamante GO. Estudo de viabilidade de utilização do material BH220 em substituição ao FEP04 em painel externo de um automóvel. In: Anais do $67^{\circ}$ Congresso ABM Internacional; 2012; Rio de Janeiro. São Paulo: ABM; 2012.

2 ULSAB-AVC Program. Technical transfer dispatch 6, 05-0I-200I, ULSAB-AVC body structures materials. Brussels: World Steel Association; 200I.

3 Chiang L-J, Yang K-C, Hsiao I-C. Effects of annealing conditions on bake hardenability for ULC steels. China: Iron and Steel Research \& Development Department China Steel Corporation; 20I I. p. I-6.

4 Ferreira FR, et al. Influência da Temperatura e do tempo de permanência no forno do aço $\mathrm{BH} 220$ no resultado de Bake Hardening. In: Anais do $68^{\circ}$ Congresso ABM Internacional; 20I3; Belo Horizonte. São Paulo: ABM; 2013.

5 Borges R. Aços com envelhecimento controlado: efeito do modo e da quantidade de pré-deformação [dissertação de mestrado]. Belo Horizonte: Universidade Federal de Minas Gerais; 1994.

6 Berbenni S, Favier V, Lemoine X, Berveiller M. A micromechanical approach to model the bake hardening effect for low carbon steels. scripta Materialia. 2004;5I (4):304. http://dx.doi.org/I0.1016/j.scriptamat.2004.04.03I.

7 Rubianes JM, Zimmer P. Proc. conf. on High Strength Sheet Steels for the Automotive Industry. Warrendale: Iron and Steel Society; 1994. p. III-II8.

8 Lora FA. Avaliação do processo de estampagem profunda de chapas de aço $\mathrm{BH} 180$ e BH 220 utilizado na indústria automobilística [dissertação de mestrado]. Porto Alegre: Universidade Federal do Rio Grande do Sul; 2009.

9 Montgomery D, Runger G. Estatística aplicada e probabilidade para engenheiros. 5. ed. Rio de Janeiro: LTC; 2013. Planejamento de experimentos com vários fatores; p. 52. cap. 14.

10 Fiat Auto Normazione. Norma 528I4: high-resistance steel sheets and strips with yeld point of I80 to 300 MPa. 3. ed. Turim: Fiat Auto Normazione; 2004.

I I Singh SB, Mohanty ON. Bake Hardening in low and medium carbon steels. Kharagpur: Indian Institute of Technology; 2012. p. I4-2I.

12 Fiat Auto Normazione. Norma 50I I3: prova di trazione: prove di carattere generico. 6. ed. Turim: Fiat Auto Normazione; 2005.

13 Fiat Auto Normazione. Norma UNIEN 10002-I: prova di trazione: método de prova a temperatura ambiente. I. ed. Turim: Fiat Auto Normazione; 2004.

14 ASTM International - ASTM. ASTM A-370: standard test methods and definitions for mechanical testing of steel products. West Conshohocken: ASTM International; 2014.

15 Lemos G. Efeito do recozimento em caixa do passe de encruamento nas propriedades mecânicas de um aço Bake Hardening. Florianópolis: Universidade Federal de Santa Catarina; 20II. p. I4- 17.

16 Mangili I, Lasagni M, Huang K, Isayev Al. Modeling and optimization of ultrasonic devulcanization using the response surface methodology based on central composite face-centered design. Chemometrics and Intelligent Laboratory Systems. 2015; I44:I-10. http://dx.doi.org/10.1016/j.chemolab.2015.03.003.

17 Kuang C, Wang J, Li J, Zhang S, Liu H, Yang H. Effect of continuous annealing on microstructure and bake hardening behavior of low carbon steel. Journal of Iron and Steel Research International. 2015;22(2): 163- 170. http://dx.doi. org/10.1016/S1006-706X(15)60025-5. 
Ferreira et al.

18 Tang Z, Ding H, Ding H, Cai M, Du L. Effect of prestrain on microstructures and properties of Si-Al-Mn TRIP steel sheet with Niobium. Journal of Iron and Steel Research International. 20 I0; I7(4):59-65. http://dx.doi.org/10.1016/ SI006-706X(10)60087-8.

Recebido em: 26 Jun. 2016

Aceito em: 9 Nov. 2016 